CASE REPORT

\title{
Lymphocytic gastritis-like T cell lymphoma: molecular evidence of an unusual recurrence
}

\author{
M E Nga, S H Tan, M Teh, E S C Koay, S M Chong, T C Putti, M Salto-Tellez
}

J Clin Pathol 2004;57:1222-1224. doi: 10.1136/icp.2004.017350

This report describes a patient with a gastric biopsy specimen showing histomorphological and immunohistochemical appearances indistinguishable from those usually present in lymphocytic gastritis, a rare condition of unknown aetiology with a distinctive phenotype. The patient had a history of a biopsy confirmed $T$ cell non-Hodgkin lymphoma at two anatomical sites (bladder and stomach), which was subsequently treated. Molecular analysis of the $\mathrm{T}$ cell receptor (TCR) $\gamma$ chain gene rearrangements showed a distinct monoclonal $T$ cell population in the bladder and gastric biopsies. The same analysis in the lymphocytic gastritis-like biopsy sample showed a monoclonal population with identical base pair size to that identified in the other specimens. This report highlights the importance of TCR gene rearrangement analysis in the diagnosis of unusual gastric inflammation, and the use of capillary electrophoresis based polymerase chain reaction in the follow up of lymphoproliferative disorders.

A 55 year old man presented with haematuria. Cystoscopy revealed a mucosal growth in the left hemitrigone and anterior wall. Bladder biopsies revealed a lymphomatous $\mathrm{T}$ cell infiltrate. The patient also complained of epigastric pain, and gastroscopy revealed a $1 \mathrm{~cm}$ incisural ulcer, surrounded by nodular mucosa. Gastric biopsy showed a $\mathrm{T}$ cell lymphoma. Thoracic and abdominal scans were negative for nodal disease. Staging bone marrow trephine biopsies revealed no tumour involvement. Treatment was instituted with the cyclophosphamide, doxorubicin, vincristine, and prednisolone regimen. Follow up cystoscopy and gastroscopy at nine months showed no gross evidence of tumour. However, the patient developed recurrent epigastric pain three months later, and gastroscopy revealed incisural scarring. Biopsy showed a lymphocytic gastritis (LG)-like picture at the cardia. There was no evidence of Helicobacter pylori infection. Molecular studies were applied to the bladder and gastric biopsies. After complete lymphoma staging (including a computed tomography scan of the thorax/ abdomen/pelvis, which was negative for lymphadenopathy, and a bone marrow examination, which histologically showed no lymphomatous involvement), a second round of chemotherapy with the ifosfamide, carboplatin, and etoposide regimen was instituted, based on the molecular findings and in view of the fact that his clinical picture was more in line with a peripheral $\mathrm{T}$ cell lymphoma; repeat gastric biopsies four months later were negative for lymphoma. The endoscopic picture of "incisural scarring" and the clinical symptoms disappeared after the second course of chemotherapy. In addition, there were no features of LG, indicating response to treatment.

\section{MOLECULAR METHODS AND RESULTS}

Immunohistochemistry for CD3, CD20, CD4, CD8, and granzyme B was performed. Dewaxed tissue sections were pretreated with Dako $^{\circledR}$ high $\mathrm{pH}$ target retrieval solution (Dako Corporation, Carpinteria, California, USA) and heated at $98^{\circ} \mathrm{C}$ for 25 minutes. The sections were then treated with $3 \%$ hydrogen peroxide solution for 10 minutes at room temperature and subsequently incubated with monoclonal antibodies for one hour. Sections were incubated with secondary antibody (antimouse or antirabbit horseradish peroxidase labelled polymer, Dako EnVision+ system) for 30 minutes, followed by the diaminobenzidine + substratechromogen solution for 10 minutes (Dako EnVision+ system). Sections were then rinsed in water, counterstained in haematoxylin, dehydrated, and mounted.

The analysis of $\mathrm{T}$ cell receptor (TCR) $\gamma$ chain gene rearrangement was performed with a fluorescence based polymerase chain reaction (PCR) method. ${ }^{1}$ Briefly, DNA was extracted using DNeasy tissue kit (Qiagen GmbH, Hilden, Germany), and the amplified PCR products for the Ja and Jp regions, ranging in size from 60 to 100 base pairs, were detected by capillary electrophoresis (CE) using an automated ABI PRISM 310 genetic analyser (Applied Biosystems, Foster City, California, USA). The sizes of the PCR products were determined using GeneScan Analysis Software ${ }^{\mathrm{TM}}$, version 3.7. The assay has a sensitivity of detecting a $1 \%$ clonal cell population in a polyclonal background.

The bladder biopsy showed mucosal and submucosal sheets of CD3 positive T cells with irregular nuclei (fig 1A). The first gastric ulcer biopsy showed, in addition to the features of an ulcer, expansion of the lamina propria by sheets of similar CD3 positive T cells (fig 1B), with some intraepithelial extension. No discernible LG-like features were identified. The post-treatment gastric incisural cardial biopsy showed the histological features of LG; namely, prominent intraepithelial lymphocytes, most highly concentrated in the gastric pits. These cells had a similar morphology to those seen in the previous biopsies, with slightly irregular nuclei and a moderate amount of pale to clear cytoplasm (fig 1C, D). A mild lymphoplasmacytic infiltrate was present in the lamina propria; however, there was no obvious expansion of the lamina propria by an atypical lymphoid infiltrate. The intraepithelial and stromal lymphocytes showed a distinctive $\mathrm{T}$ cell (CD3 and CD8 positive; CD20, CD4, and granzyme B negative) immunophenotype. Figure 2 shows the results of the TCR $\gamma$ chain gene rearrangement analysis. Analysis of the bladder biopsy, the first gastric biopsy, and the follow up LG-like biopsy showed similar findings; namely, that of a single, 68 base pair peak, corresponding to a monoclonal $\mathrm{T}$ cell population. This precise base pair size was identified in all three biopsy specimens. Furthermore, the sensitive CE detection method revealed no

Abbreviations: LG, lymphocytic gastritis; PCR, polymerase chain reaction; TCR, T cell receptor 

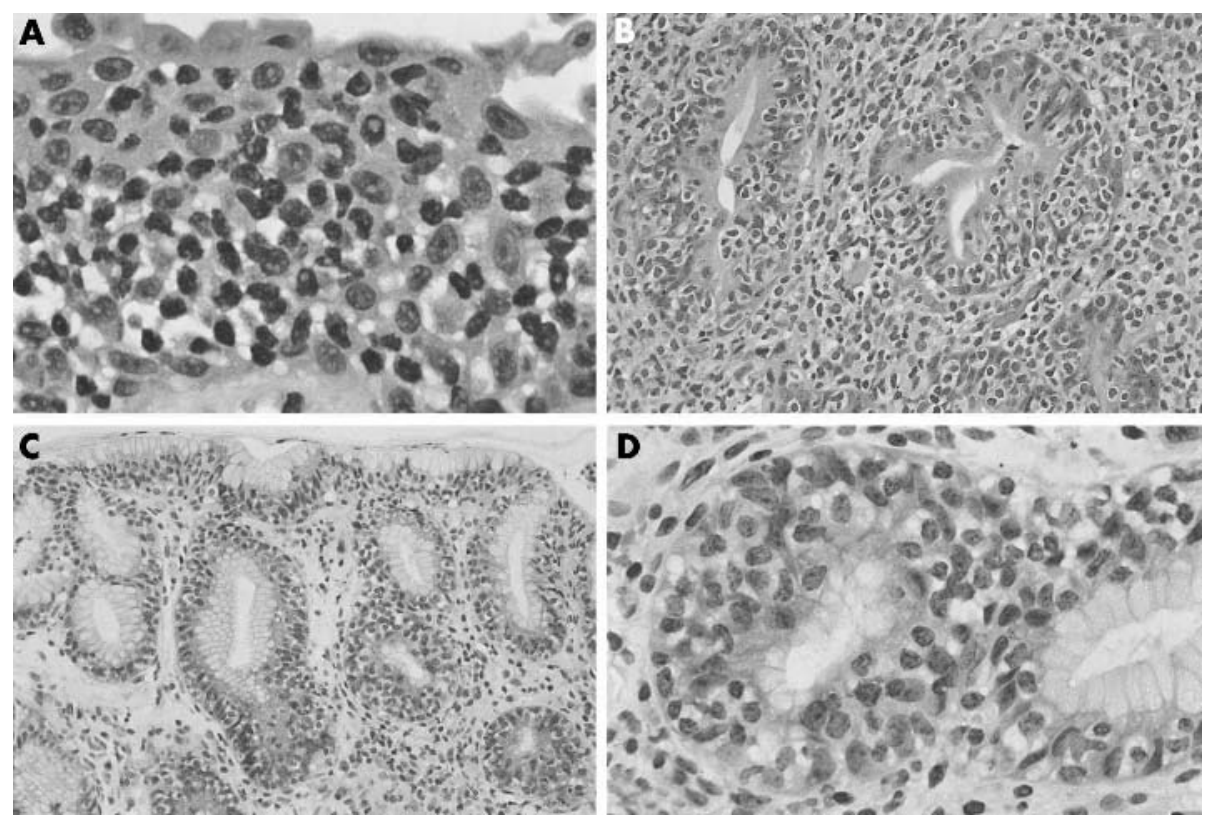

Figure 1 (A) Bladder biopsy showing sheets of atypical lymphoid cells infiltrating the mucosa and submucosa (haematoxylin and eosin (H\&E) stain; original magnification, $\times 75$ ). (B) Initial gastric ulcer biopsy showing lamina propria expansion by the neoplastic atypical T lymphocytes (H\&E stain; original magnification, $\times 30$ ). (C) Follow up gastric cardia biopsy showing a lymphocytic gastritis type picture with prominent atypical lymphocytes situated in the gastric epithelium (H\&E stain; original magnification, $\times 20$ ). (D) High power view of the lymphocytic gastritis-like appearance of the follow up biopsy (H\&E stain; original magnification, $\times 60)$.

accompanying polyclonal $\mathrm{T}$ cell population in any of the three samples, indicating that most of the $\mathrm{T}$ cells present in the three specimens belong to the same neoplastic clone. To confirm this, the PCR product of the three analyses was sequenced and, within the limitations of the sample size, the results were identical (data not shown). A similar analysis in a bona fide lymphocytic gastritis case was performed as a parallel control, and showed a polyclonal pattern.

\section{DISCUSSION}

$\mathrm{LG}^{23}$ is a form of inflammatory infiltration of the gastric mucosa almost exclusively composed of lymphocytes. Endoscopically, it is characterised by a combination of superficially eroded and nodular mucosal areas. The histological appearance is that of a mixture of superficial erosions and distinctive small, mature lymphocytes within the surface epithelium, of T cell type. The aetiology is unknown, although associations have been postulated with coeliac disease, ${ }^{4}$ protein loss enteropathy, ${ }^{5}$ and H pylori infection.

"The use of molecular tests for clonality studies in gastric lesions is almost exclusively confined to the analysis of B cell lymphoproliferative disorders"

We present a case with the morphological features of LG, but with molecular evidence of $\mathrm{T}$ cell lymphoma, in a patient with a history of $\mathrm{T}$ cell lymphoma.

The use of molecular tests for clonality studies in gastric lesions is almost exclusively confined to the analysis of B cell lymphoproliferative disorders, ${ }^{6}{ }^{7}$ particularly in the context of dense $H$ pylori infection, and thus based on $\operatorname{IgH}$ gene rearrangement analysis. Here, TCR gene rearrangement molecular tests were applied to a gastric biopsy. The case was phenotypically indistinguishable from LG, a benign condition of unknown aetiology, and yet genotypically it was shown to represent a recurrence of the patient's $\mathrm{T}$ cell lymphoma. Most primary gastric lymphomas are of B cell origin, often derived from the mucosa associated lymphoid
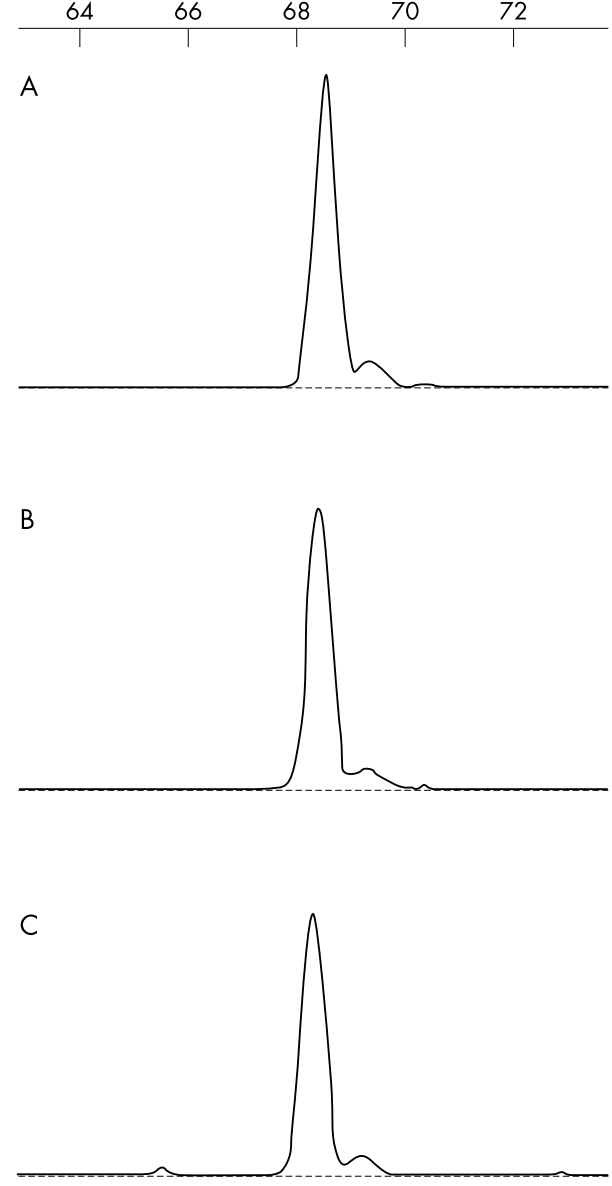

Figure 2 T cell receptor gene rearrangement analysis by fluorescence polymerase chain reaction and capillary electrophoresis detection. Note the presence of the same monoclonal T cell population in the three samples analysed: (A) bladder biopsy; (B) original gastric biopsy; and (C) lymphocytic gastritis-like biopsy. See text for further comments. 


\section{Take home messages}

- We describe a patient with a history of T cell lymphoma at two sites in whom a gastric biopsy had the appearance of lymphocytic gastritis (LG), a benign condition

- However, the original lymphomas showed a monoclonal pattern of T cell receptor (TCR) $\gamma$ chain gene rearrangement and the same pattern was found in the LG-like lesion

- Thus, TCR gene rearrangement analysis can play an important role in the diagnosis of unusual gastric inflammation and in the follow up of lymphoproliferative disorders

tissue, and primary T cell gastric lymphomas are rare. ${ }^{8}$ In the context of a patient with known extranodal $\mathrm{T}$ cell lymphoma, it is essential to characterise all lymphoid lesions, to prove or exclude a common clonal origin with the known primary. This approach helps in understanding the extent, distribution, treatment response, and natural history of rare $\mathrm{T}$ cell lymphoproliferative lesions affecting multiple extranodal sites, either synchronously or metachronously. This has direct impact on the management and prognostication of this disease, as highlighted by our case.

To date, T cell lymphoma appearing morphologically as an LG-like lesion has not been described. This could be because of the rarity of these lesions and the consequent lack of clinicopathological and molecular characterisation. This case highlights the importance of TCR gene rearrangement studies in diagnosis in an otherwise histologically uninformative gastric biopsy that only shows the features of LG. It presents a unique situation where a neoplastic condition possesses the histological features of LG, which has not been previously associated with malignant conditions. Further studies need to be done to characterise LG on a molecular and immunophenotypical level.

\section{Authors' affiliations}

M E Nga, M Teh, E S C Koay, S M Chong, T C Putti, M Salto-Tellez, Department of Pathology, National University of Singapore, 5 Lower Kent Ridge Road, Singapore 119074

S H Tan, Department of Haemato-Oncology, National University Hospital, Singapore

E S C Koay, M Salto-Tellez, Molecular Diagnosis Centre, Department of Laboratory Medicine, National University Hospital, Singapore

Correspondence to: Dr M Salto-Tellez, Department of Pathology, National University of Singapore, 5 Lower Kent Ridge Road, Singapore 119074; patmst@nus.edu.sg

Accepted for publication 7 April 2004

\section{REFERENCES}

1 Chang TL, Salto-Tellez M, Thamboo TP, et al. Diagnostic validation of capillary electrophoresis analysis of T-cell receptor gamma-chain gene rearrangements: prediction of malignant transformation of cutaneous T cell lymphoproliferative disorders. Clin Chem 2003:49:513-15.

2 Haot J, Hamichi L, Wallez L, et al. Lymphocytic gastritis: a newly described entity: a retrospective endoscopic and histological study. Gut 1988:29:1258-64.

3 Rubio CA, Kato Y, Sugano $\mathrm{H}$. The lymphoepithelial phenomenon in the gastric mucosa. Pathol Res Pract 1985;180:612-14

4 Wolber R, Owen D, DelBuono L, et al. Lymphocytic gastritis in patients with celiac sprue or sprue-like intestinal disease. Gastroenterology 1990:98:310-15.

5 Wolber RA, Owen DA, Anderson FH, et al. Lymphocytic gastritis and giant gastric folds associated with gastrointestinal protein loss. Mod Pathol 1991;4:13-15.

6 Sukpanichnant S, Vnencak-Jones CL, McCurley TL. Determination of B cell clonality in paraffin-embedded endoscopic biopsy specimens of abnormal lymphocytic infiltrates and gastrointestinal lymphoma by polymerase chain reaction. Am J Clin Pathol 1994;102:299-305.

7 Wundisch T, Neubauer A, Stolte M, et al. B-cell monoclonality is associated with lymphoid follicles in gastritis. Am J Surg Pathol 2003;27:882-7.

8 Niitsu N, Nakamine H, Kohri M, et al. Primary gastric T-cell lymphoma not associated with human T-lymphotropic virus type l: a case report and review of the literature. Ann Hematol 2003;82:197-202. 